\title{
BMJ Open Patient engagement in the development of best practices for transitions from hospital to home: a scoping review
}

\author{
Grace Zhao, ${ }^{\oplus 1}$ Carol Kennedy, ${ }^{2}$ Gracia Mabaya, ${ }^{2}$ Karen Okrainec, ${ }^{3}$ Tara Kiran $^{2,4,5}$
}

To cite: Zhao G, Kennedy C, Mabaya G, et al. Patient engagement in the development of best practices for transitions from hospital to home: a scoping review. BMJ Open 2019;9:e029693. doi:10.1136/ bmjopen-2019-029693

- Prepublication history and additional material for this paper are available online. To view these files, please visit the journal online (http://dx.doi. org/10.1136/bmjopen-2019029693).

Received 6 February 2019 Revised 13 June 2019 Accepted 14 June 2019

Check for updates

(C) Author(s) (or their employer(s)) 2019. Re-use permitted under CC BY-NC. No commercial re-use. See rights and permissions. Published by BMJ.

${ }^{1}$ MD Program, Faculty of Medicine, University of Toronto, Toronto, Ontario, Canada ${ }^{2}$ Quality Standards, Evidence Development and Standards, Health Quality Ontario, Toronto, Ontario, Canada

${ }^{3}$ Department of Medicine, University Health Network and University of Toronto, Toronto, Ontario, Canada

${ }^{4}$ Department of Family and Community Medicine, St.

Michael's Hospital and

University of Toronto, Toronto,

Ontario, Canada

${ }^{5}$ Centre for Urban Health

Solutions, Li Ka Shing

Knowledge Institute, Toronto, Ontario, Canada

Correspondence to

Ms Grace Zhao;

grace.zhao@mail.utoronto.ca

\section{ABSTRACT}

Objectives To explore the extent of patient engagement in the development of best practice reports related to transitions from hospital to home.

Design Scoping review.

Data sources Electronic databases (MEDLINE, EMBASE, CINAHL, Scopus, Trip Database, DynaMed Plus and Public Health Plus) and multiple provincial regulatory agency and healthcare organisation websites.

Eligibility criteria We included best practice reports related to the transition from hospital to a long-term care facility, community dwelling or rehabilitation centre. We included documents disseminated in English between 1947 and 2019.

Data extraction and synthesis Two independent reviewers screened for eligibility and one extracted and analysed data using a data extraction tool we developed based on established patient engagement frameworks. Only records actively engaging patients were analysed $(n=11)$. The methodological quality of actively engaging patients was assessed using domain 2 (item 5) of stakeholder involvement from the Appraisal of Guidelines for Research and Evaluation II (AGREE II) tool.

Results The search yielded 1921 citations of which 23 met the inclusion criteria and were included for narrative synthesis. These were disseminated between 1995 and 2019, with 18 (78\%) published after 2010. Most were conducted in North America (USA 43\%, Canada 22\%), Europe (UK 30\%) and Australia (4\%). Eleven (48\%) actively involved patients, of which only two involved patients across all stages of development. Most involved patients through direct or indirect consultation. The mean AGREE II domain 2 item 5 score (of those that actively engaged patients) was 5.9 out of 7 .

Conclusions Only half of existing best practice reports related to the transition from hospital to home actively involved patients in report development. However, the extent of patient engagement has been increasing over time. More organisations should strive to engage patients throughout the best practice development process and provide patients with opportunities for shared leadership.

\section{INTRODUCTION}

Around the world, healthcare systems strive towards patient-centred care. ${ }^{1-4}$ In the UK, patients have been involved in health research for at least 15 years $^{56}$ and patient engagement is a prerequisite for many funding bodies

\section{Strengths and limitations of this study}

- We conducted a scoping review of both peer-reviewed and grey literature to identify and assess relevant best practice reports including quality standards, clinical guidelines and consensus statements.

- We used existing patient engagement frameworks to develop a data extraction tool that specifically assessed the level of patient engagement from consultation to involvement to shared leadership.

- We used item 5, domain 2 from the Appraisal of Guidelines for Research and Evaluation II reporting checklist to assess the extent to which patient and public perspectives were sought during best practice report development.

- Research was done in partnership with a health quality agency preparing to inform patient engagement in the development of a quality standard on the transition from hospital to home.

- Due to limited documentation, some patient engagement activity may not have been reported which may mask actual level of patient engagement.

and research ethics approval. ${ }^{6}$ In the USA, patient engagement was mandated in health research processes once the Patient Centered Outcomes Research Institute was established in $2010 .{ }^{58}$ The Canadian Institutes of Health Research created the Strategy for Patient-Oriented Research patient engagement framework in $2014.9^{9}$ Increased involvement of patients in health services research, along with engagement in healthcare delivery and policy-making, has led to a "patient revolution' in the last few decades. ${ }^{4810-12}$

Patient engagement in clinical practice guideline development has been recommended by multiple institutions including the National Institute for Clinical Excellence (NICE),${ }^{13}$ the $\mathrm{WHO}^{14}$ and the Guidelines International Network. ${ }^{15}$ Even guideline appraisal tools, such as the Appraisal of Guidelines for Research and Evaluation II (AGREE II) has a specific section for the evaluation of patient engagement. ${ }^{16}$ Despite this increase in patient engagement, there is a lack of consensus on 
how and when to optimally engage patients. ${ }^{4}$ Further, it is unclear how widely organisations follow the patient engagement guidance in the development of guidelines or other evidence-based recommendations.

The transition from hospital to home is an important, yet vulnerable exchange point that if not properly handled can lead to adverse clinical events and preventable hospital readmissions. ${ }^{1718}$ Often patients are not involved in their care transitions planning, ${ }^{18-21}$ which can result in unmet needs once home. ${ }^{22}{ }^{23}$ Understanding the patient's perspective is important in not only meeting their needs postdischarge but also in improving health outcomes. ${ }^{1824}$ Patient and caregiver involvement in guidance development may address some of these known gaps in care.

Currently, little is known about the extent to which patients and caregivers have been involved in the development of best practice reports related to the transition from hospital to home. Hence, we conducted a scoping review to assess the extent of patient engagement in the development of best practice reports on transitions from hospital to home. We define best practice reports as documents that provide evidence-based recommendations for providing quality care on a particular medical condition or topic. We used Carman $e t a l$ s patient engagement framework because it examines the continuum of engagement at the policy-making level from consultation or involvement to partnership and shared leadership. ${ }^{24}$ We focus on active patient engagement, defined here as participation in an advisory or working group and/or direct consultation whereby resultant discussions informed the content of the report. ${ }^{25}$ Our findings would inform the approach to patient engagement in the development of a quality standard in transitions by the provincial quality agency in Ontario, Health Quality Ontario (HQO).

\section{METHODS}

We conducted a scoping review with guidance from the scoping review methodological framework developed by Arksey and O'Malley ${ }^{26}$ as well as from PRISMA Extension for Scoping Reviews (PRISMA-ScR) guidelines. ${ }^{27}$ The protocol was developed through consultation with the project team, including a health sciences information specialist, a clinician investigator and quality improvement professionals from HQO who are involved in the development of quality standards. Details of the protocol can be requested from the corresponding author.

\section{Inclusion criteria}

We aligned our inclusion criteria to match the anticipated scope of HQO's quality standard on transitions of care. We included best practice reports (ie, clinical guidelines, quality standards, consensus statements, guiding principles, best practice recommendations) related to the transition from hospital to home. Home was broadly defined as a person's place of residence for an extended period of time (ie, long-term care facilities, skilled nursing facilities, community dwellings (ie, retirement home, house, apartment, shelter) or rehabilitation centres). Note that rehabilitation centres were included because HQO's patient and family advisors had suggested to include this along with complex continuing care centres as part of the broad definition of home. We included all populations (ie, disease/conditions, ages, ethnicity, gender). We included documents disseminated in English between 1947 and 2019 published in either the peer-reviewed or grey literature. We had no exclusion by year of publication as we found that literature on patient engagement in the creation of guidelines is quite limited. Foreign language was excluded because of cost and time involved in translating materials.

\section{Search strategy}

We developed a search strategy in consultation with an information specialist (online supplementary appendix A). She devised an initial search strategy which was later refined in light of early results. She searched seven electronic databases covering sources from 1947 to May 16, 2018: MEDLINE (OVID), EMBASE (OVID), CINAHL (EBSCO), Scopus, Trip Database, DynaMed Plus and Public Health Plus. The updated search was conducted on 7 May 2019 (online supplementary appendix B). Electronic search terms included but were not limited to 'transitional care,' 'guidelines,' 'best practices,' 'consensus,' 'guiding principles' and 'standards'. With consultation from HQO, we identified provincial healthcare quality councils and major international organisations that we hypothesised would hold the majority of grey literature on best practice reports. The updated grey literature search was informed by extensive, iterative searches in Google to identify organisations that create or study health guidelines, as well as a federated search in MacPlus, McMaster University's Health information unit. We searched website content using keywords such as 'transition', 'transition care' or transitional care. Each website was searched for primary and secondary literature.

\section{Selection of sources of evidence}

We conducted and reviewed peer-reviewed and grey literature searches separately. Search results were imported into an online systematic review software, Rayyan. ${ }^{28}$ First, titles and abstracts were reviewed to determine eligibility for full-text review. Screening was performed blindly by two independent reviewers, with one reviewer (GZ) reviewing all titles and abstracts. Any discrepancies between reviewers were discussed and consensus was reached. We conducted a pilot screen of 20 articles which were reviewed among all members of the project team to test the reviewer software and address any concerns with the article selection criteria developed a priori. From those selected at title and abstract screening, one reviewer (GZ) further reviewed the full-text records for eligibility owing to resource limitations. 


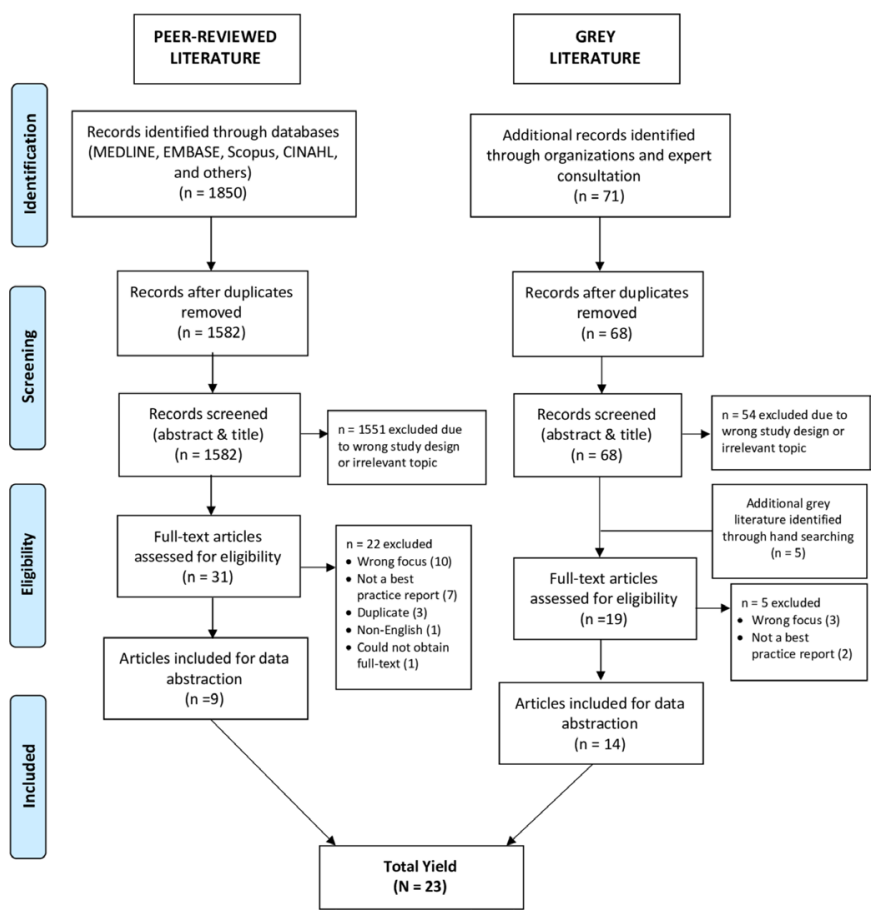

Figure 1 PRISMA flow diagram of literature search and selection process. PRISMA, Preferred Reporting Items for Systematic Reviews and Meta-Analyses.

\section{Data extraction and synthesis}

GZ performed the data extraction using a data extraction tool (online supplementary appendix $\mathrm{C}$ ). The data extraction tool was developed based on published patient engagement frameworks ${ }^{92429}$ and tested by the team before use. We used Carman $e t a l$ s patient engagement framework ${ }^{24}$ to determine the types and extent of patient engagement. We focused on the level of engagement at the policy-making level, and considered the continuum of engagement (consultation, involvement and shared leadership). For included articles, GZ extracted report characteristics (ie, year, jurisdiction, literature type), whether patients were involved, terminology, whether consideration was given to marginalised populations, and specifics of when and how patients were involved. For simplicity, here 'patients' refer to patients and caregivers. Particular attention was paid to whether patients were participants of the advisory or working group that developed the recommendations. We restricted our analysis to records actively engaging patients $(n=10)$. Data will be presented in a narrative format with tables and figures.

\section{Quality appraisal}

Quality appraisal of the best practice reports was performed using one item from the AGREE II reporting checklist. ${ }^{30}$ We used item 5 from domain II (stakeholder involvement) to assess whether the perspectives of the target population (ie, patients, public and so on) were sought. The item is scored out of 7 , where a score of 1 indicates that there is no information that is relevant to the AGREE II item or if the concept is very poorly reported, and a score of 7 indicates exceptional quality
Table 1 Overview of selected record characteristics

\begin{tabular}{|c|c|}
\hline $\begin{array}{l}\text { Characteristic } \\
(n=23)\end{array}$ & Output \\
\hline Year & $\begin{array}{l}1995-2000(13 \%, n=3) \\
2000-2009(9 \%, n=2) \\
2010-2018(78 \%, n=18)\end{array}$ \\
\hline Record type & $\begin{array}{l}\text { Guidelines document }(43 \%, n=10) \\
\text { Consensus statement }(22 \%, n=5) \\
\text { Best practice recommendations }(17 \%, n=4) \\
\text { Other }(17 \%, n=4)\end{array}$ \\
\hline Geography & $\begin{array}{l}\text { USA }(43 \%, n=10) \\
\text { UK }(30 \%, n=7) \\
\text { Canada }(22 \%, n=5) \\
\text { Australia }(4 \%, n=1)\end{array}$ \\
\hline Jurisdiction & $\begin{array}{l}\text { Government agency }(30 \%, n=7) \\
\text { Professional organisation }(30 \%, n=7) \\
\text { Non-profit organisation }(17 \%, n=4) \\
\text { Other }(22 \%, n=5)\end{array}$ \\
\hline Topic area & $\begin{array}{l}\text { General }(52 \%, n=12) \\
\text { Stroke }(17 \%, n=4) \\
\text { Heart failure }(17 \% n=4) \\
\text { Neurological }(4 \%, n=1) \\
\text { End of life care }(4 \%, n=1) \\
\text { Chronic obstructive pulmonary disease }(4 \%, n=1)\end{array}$ \\
\hline Active PE & $\begin{array}{l}\text { Yes }(48 \%, n=11) \\
\text { No }(52 \%, n=12)\end{array}$ \\
\hline
\end{tabular}

$\mathrm{PE}$, patient engagement.

of reporting and where the full criteria articulated in the reporting checklist have been met.

\section{Patient and public involvement}

Patients and the public were not involved in the development of this study.

\section{RESULTS}

The systematic search yielded 1582 peer-reviewed and 68 grey literature results after duplicates were removed (figure 1). Overall, 1605 articles were excluded at the title and abstract screen. A further 22 peer-reviewed and 5 grey literature were excluded for the following reasons: 23 did not meet the inclusion criteria, 3 were duplicates within the peer-reviewed searches and 1 full-text article could not be obtained. The remaining 23 relevant reports from peer-review $(n=9)$ and grey literature $(n=14)$ met our relevance screen.

\section{Report characteristics}

The included 23 records were published between 1995 and 2018, with 18 (78\%) published in 2010 and beyond (table 1 ). Of these, 10 were guidelines, 5 were consensus statements, 4 were best practice recommendations, 2 were guides, 1 was a discharge care bundle and another was a scientific statement. Most were conducted in North America $(n=15 ; 65 \%)$ and the UK $(n=7 ; 30 \%)$. Only 10 of the best practice reports considered marginalised populations in their search and/or assessment of recommendations (table 2). Eleven (48\%) out of 23 


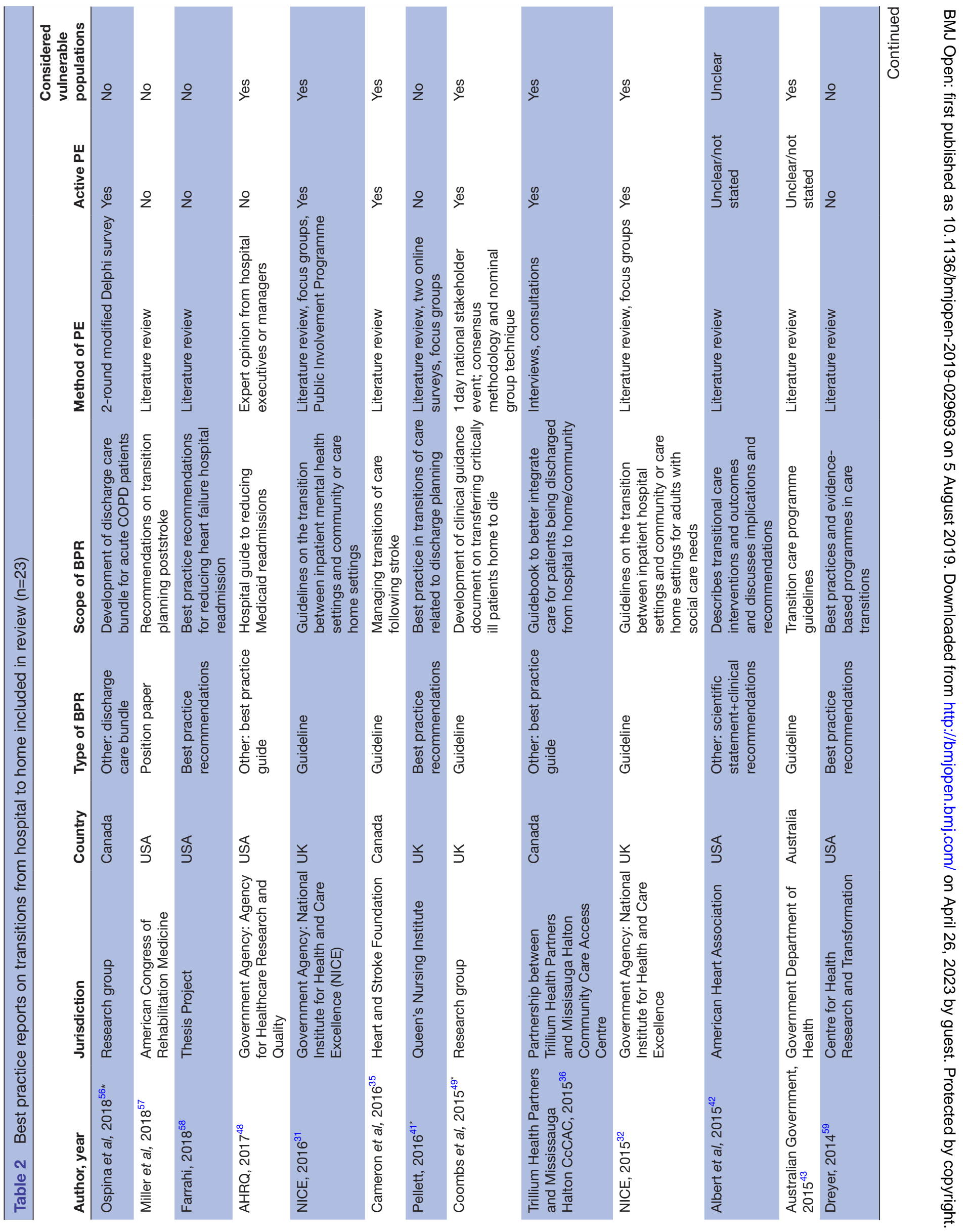




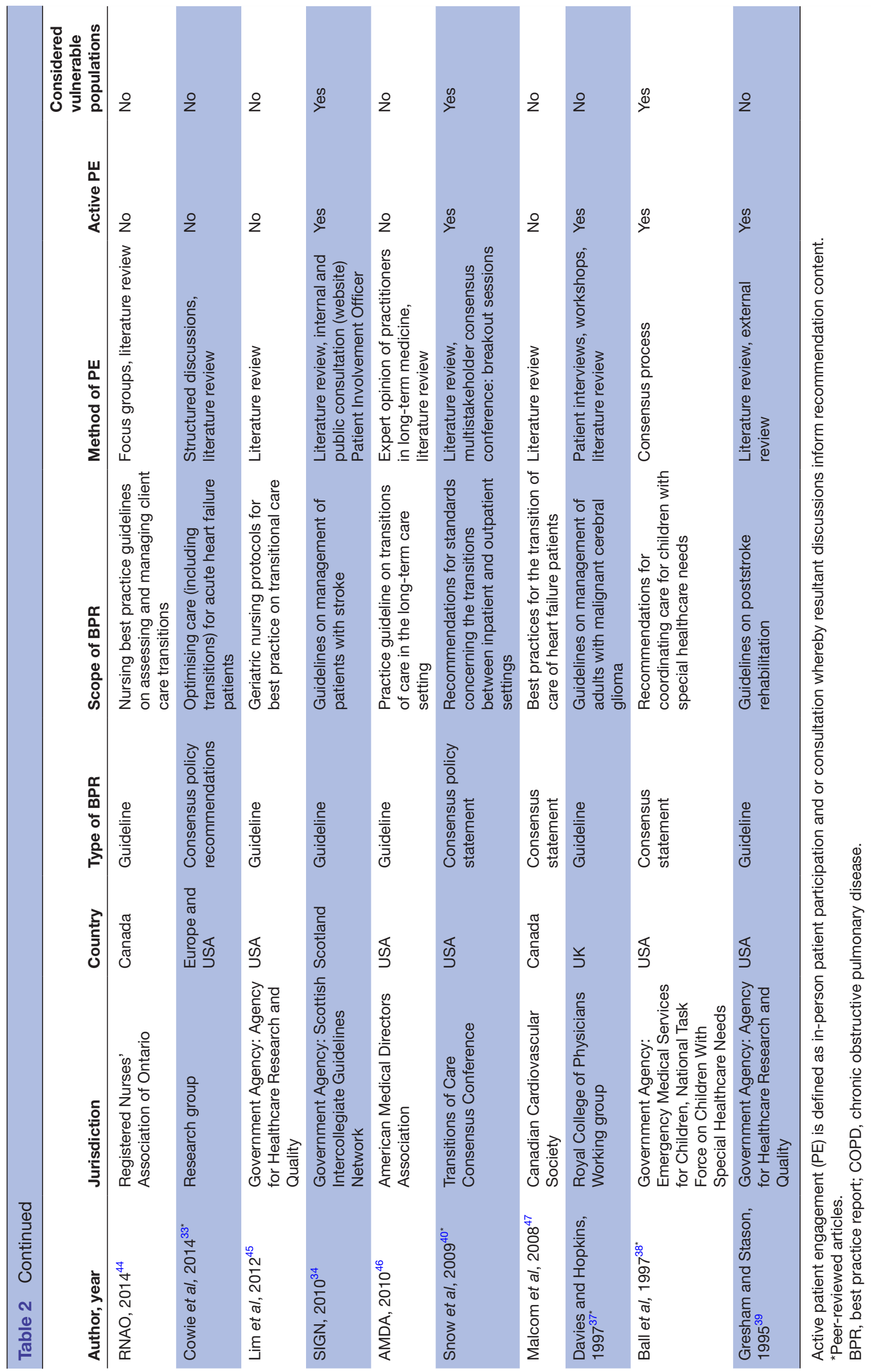


records actively engaged patients in the development of the best practice reports.

\section{Types of patient engagement}

Using Carman et als patient and family engagement framework, ${ }^{24}$ we found that when patients were engaged, most were engaged in the consultation (direct and indirect) stage, some in involvement, and a few in shared leadership (table 3). Direct consultation included surveys, an open forum, patient interviews, workshops, conference breakout sessions, focus groups and peer review of provisional guidelines. Indirect consultation included four instances where patient preferences and values informed the literature search. ${ }^{31-34}$ Involvement activities included eight instances where patients were involved in the guideline developing group. ${ }^{31-33}$ 35-39 Shared leadership included one instance where a patient was acknowledged as a coauthor $^{35}$ and two instances where patients were involved from the outset..$^{34}$

Item 5 assesses whether the views and preferences of the target population were considered. Score ranges from 1 (no patient engagement (PE)) to 7 (maximum PE).

\section{Quality appraisal}

Of the records that actively engaged patients $(n=11)$, the mean quality score for reporting of patient engagement was 5.9 out of 7 based on the AGREE II tool (domain 2, item 5) (table 4). The quality scores ranged from 4 to $7(\mathrm{SD}=1.04)$, with the best practice reports from 2010 onwards scoring either 6 or 7 ( $\mathrm{SD}=0.53)$. Two of the four rating criteria-statement of patient engagement strategy type and methods by which preferences and views were sought-were met by all eleven of those reports that included patient engagement in their development. The type of information gathered and how it was used to inform the development process was less often addressed in records published between 1995 and 2009.

\section{Level of patient engagement}

In earlier years (1995-2009), patient engagement primarily occurred in the post-development stage, after provisional guidelines had been drafted. In three reports within this time period, patients discussed and debated provisional guidelines either at a conference breakout session, a workshop, or an open forum. ${ }^{37} 3940$ During this period, patients were minimally represented in the working group that writes the guidelines (developing stage), oftentimes having as little as one patient representative on a committee of up to 33 professionals. ${ }^{38} 39$ Many of the recommendations were developed by experts and medical professionals only and did not involve patients. ${ }^{33} 40-47$ Starting in 2010, a greater proportion of the working group was comprised of people with lived experiences ${ }^{3132} 34-36$ and considered the patient perspective when searching the literature. ${ }^{31} 3234$

Within each stage of development, the precise contributions of patients and caregivers were unclear.
In one paper, ${ }^{38}$ there was little information on how the consumer representative (1 of 33 professionals) was involved in the task force as the authors did not elaborate on the methodology of developing the recommendations. For the development of the Transitions of Care Consensus Policy Statement, ${ }^{40}$ a consensus conference was convened which had attendees representing patient groups. However, it was not stated how many participants represented the patient groups nor were individual patients acknowledged in the manuscript.

\section{UK government agencies did better}

The best practice reports developed in the UK, SIGN and NICE involved patients at all stages of the development process and had a greater proportion of patients sitting in the working group. ${ }^{31} 32{ }^{34}$ Furthermore, more UK best practice reports considered marginalised populations. Of the 10 $10^{31} 3234-363840434849$ reports that had considered this, $4^{31323449}$ were developed from the UK.

As part of the National Health Service, both SIGN and NICE have to complete an 'equality impact assessment' to identify and address any equality issues regarding age, disability, gender, race, religion and/or sexual orientation. ${ }^{50}$ For example, NICE's 2015 guideline on the transition between inpatient hospital settings and community settings for adults with social care needs had special considerations for the homeless and lesbian, gay, bisexual, trans and queer (LGBT) populations.

\section{DISCUSSION}

We found that only half of best practice reports related to the transitions from hospital to home actively involved patients in report development. The reporting on the level of patient engagement was generally good, although some reports lacked detail about the exact contributions of patients. Overall, there has been an increase over time in the amount of patient engagement in the development of best practice reports for the transition from hospital to home. Since 2010, patients have been engaged earlier in the planning stages and also have been more involved as participants of working groups and advisory committees. Few organisations involved patients in shared leadership with only two organisations that involved patients at every stage of development from guideline topic proposal to peer review.

Overall, UK government agencies, such as the NICE and SIGN, were more advanced at engaging patients throughout the development process, had clearer documentation of their involvement and did better at considering marginalised populations. This is unsurprising as reporting of patient engagement has been required in the UK for over 15 years, much longer than other countries, and also reflects the cultural shift towards the importance of engaging and reporting patient engagement. ${ }^{6}{ }^{51} \mathrm{UK}$ government agencies deliberately factor patient engagement in the development of these reports by having 'patient involvement officers' or a 'public involvement 
Table 3 Reporting of patient engagement for included studies with active patient engagement $(n=11)$

\begin{tabular}{|c|c|c|c|}
\hline Reference & Consultation & Involvement & Shared leadership \\
\hline $\begin{array}{l}\text { Ospina et al, } \\
2018^{56}\end{array}$ & $\begin{array}{l}34 \text { COPD patients were involved in } \\
\text { survey to inform the development of } \\
\text { the COPD discharge bundle. }\end{array}$ & None. & None. \\
\hline $\begin{array}{l}\text { Cameron et al, } \\
2016^{35}\end{array}$ & $\begin{array}{l}\text { At least } 2 \text { patients or caregivers were } \\
\text { external reviewers. }\end{array}$ & $\begin{array}{l}\text { Stroke survivor(s) were part of the writing } \\
\text { group which discussed and debated the } \\
\text { value of evidence of recommendations. }\end{array}$ & $\begin{array}{l}\text { One stroke survivor was made a } \\
\text { coauthor of the journal article. }\end{array}$ \\
\hline NICE, $2015^{32}$ & $\begin{array}{l}\text { Public and patient involvement } \\
\text { programme (PPIP) provides input on } \\
\text { provisional guidelines. } \\
\text { Patient preferences and values } \\
\text { considered in developing research } \\
\text { questions. }\end{array}$ & $\begin{array}{l}4 \text { patients and caregivers were part of the } \\
\text { guideline committee }(4 / 14) \text {. } \\
1 \text { PPIP member sits on the scoping } \\
\text { group. }\end{array}$ & \\
\hline
\end{tabular}

Coombs et al, $2015^{49}$
Patients (unclear how many) engaged None. in focus groups that developed questions to be used in the national stakeholder meeting to inform the development of a clinical guidance document on transferring critically ill patients home to die.

- At the meeting, these patients were also involved in informing guidance content by participating in the event's activities.

\begin{tabular}{|c|c|c|c|}
\hline Network, $2010^{34}$ & $\begin{array}{l}\text { Patient involvement officer provided } \\
\text { support and facilitation for the } \\
\text { guideline development group (GDG). } \\
\text { Draft guideline was available on the } \\
\text { SIGN website for a month to allow the } \\
\text { public to comment. } \\
\text { At least } 2 \text { peer reviewers were } \\
\text { patients or caregivers. } \\
\text { Members of the SIGN patient network } \\
\text { were also invited to comment on the } \\
\text { draft guideline. }\end{array}$ & $\begin{array}{l}4 \text { lay representatives were part of } \\
\text { the GDG; at least } 2 \text { were patient } \\
\text { representatives selected from } \\
\text { national and/or local patient-focused } \\
\text { organisations in Scotland }(4 / 26) \text {. } \\
\text { Representatives participated in informal } \\
\text { consensus. }\end{array}$ & $\begin{array}{l}\text { Patients were engaged at every } \\
\text { stage. Individuals or patient } \\
\text { groups may propose a guideline } \\
\text { topic through an application. }\end{array}$ \\
\hline $\begin{array}{l}\text { Snow et al, } \\
2009^{40}\end{array}$ & $\begin{array}{l}\text { Patient groups from the Institute } \\
\text { for Family Centred Care attended } \\
\text { the Transitions of Care Consensus } \\
\text { Conference (unclear how many). } \\
\text { Patient groups were involved in } \\
\text { breakout sessions focused on } \\
\text { discussing the principles and } \\
\text { standards already drafted for revision. } \\
\text { Revision, refinement and prioritisation } \\
\text { of standards were done through a } \\
\text { group consensus voting process. }\end{array}$ & None. & None. \\
\hline Ball et al, $1997^{38}$ & None. & $\begin{array}{l}\text { One patient representative was a part } \\
\text { of the multidiscipline task force that } \\
\text { developed the transitional care plan } \\
(1 / 33) \text {. } \\
\text { Unclear on the consensus process. }\end{array}$ & None. \\
\hline
\end{tabular}


Table 3 Continued

\begin{tabular}{|c|c|c|c|}
\hline Reference & Consultation & Involvement & Shared leadership \\
\hline $\begin{array}{l}\text { Davies and } \\
\text { Hopkins, } 1997^{37}\end{array}$ & $\begin{array}{l}\text { Patients were interviewed to inform } \\
\text { guideline for patient management } \\
\text { concerning communication and } \\
\text { support. } \\
\text { Patients were involved with approval } \\
\text { of final guidelines. }\end{array}$ & $\begin{array}{l}\text { Patient and relative groups (unclear how } \\
\text { many) were a part of the working group, } \\
\text { which reviewed and debated provisional } \\
\text { guidelines derived from research } \\
\text { literature. }\end{array}$ & None. \\
\hline $\begin{array}{l}\text { Gresham } \\
\text { and Stason, } \\
1995^{39}\end{array}$ & $\begin{array}{l}\text { The public was consulted through } \\
\text { an open forum where professional } \\
\text { and provider organisations, } \\
\text { manufacturers, pharmaceutical firms } \\
\text { and individuals can present written or } \\
\text { oral statements. } \\
\text { Patient organisations reviewed the } \\
\text { patient and family guide. }\end{array}$ & $\begin{array}{l}\text { Guidelines are developed by a } \\
\text { multidisciplinary panel of experts with } \\
\text { one patient representative who provided } \\
\text { the patient perspective }(1 / 16) \text {. }\end{array}$ & None. \\
\hline
\end{tabular}

programme'. ${ }^{31} 3234$ Additionally, SIGN allows any patient or group to propose a guideline topic and writes to various patient organisations and National Health Service organisations prior to the first meeting of the guideline development group to solicit input. The patient engagement officers encourage patient participation throughout all stages of development and provide training for all stakeholders involved. This addresses some of the barriers to patient engagement implementation, such as lack of organisational support, limited resources (space and time), resistance to the idea of power sharing, and perceived importance of patient engagement. ${ }^{4} 813$ 51-54 However, Canadian jurisdictions have more recently been engaging patients earlier, notably in the example of the Seamless Transitions: Hospital to Home initiative where patients were engaged throughout the design process. ${ }^{36}$

While recent literature on patient engagement focuses on hospital service improvement ${ }^{53}$ or health services research, ${ }^{6}{ }^{12}$ our paper highlights the extent of patient engagement in best practice reports focused on transitions from hospital to home. The findings of this review are consistent with earlier studies on patient engagement in health services research, planning and design. More often than not, there was little detail on the precise contributions of the patient representatives within the working group, which seems to be consistent with the literature. ${ }^{65355}$ Some reasons for this include the complexity of patient engagement being a multifaceted social process ${ }^{7}$ and that journals and peer reviewers do not usually request patient engagement information within manuscripts until more recently. ${ }^{6}$ However, we found that in recent years, patient engagement documentation has become clearer and easier to find with respect best practice reports, particularly those developed by government agencies in the UK.

The strengths of this review include a comprehensive systematic search which included searching the peer-reviewed and grey literature, the use of several patient engagement frameworks to develop our data extraction tool, and compliance with standards for the conducting and reporting of reviews (Preferred Reporting Items for
Systematic Reviews and Meta-Analyses). There were also some notable limitations. With respect to article selection, an additional reviewer to screen the full-text articles could have increased the rigour of the selection process. This was mitigated by sharing all included and excluded articles with documentation of reasons for exclusion to the project members. Owing to resource limitations, only one reviewer performed the data extraction, and thus it is possible that some information may have been missed. Some patient engagement activity may not have been reported in the publication. Further, we did not solicit information from authors of the best practice reports to confirm. Although the type of patient engagement was captured in all of the studies, we do not know how influential their contributions were.

Our findings highlight the opportunity for organisations to better engage patients in any quality improvement initiative. Five strategies that organisations can consider are (1) engage patients in developing the scope of the problem, (2) involve patients in the writing of recommendations, (3) consider the needs of vulnerable populations, (4) use a patient engagement officer to recruit patients to participate across all stages of development and (5) document patients' precise contributions. Many of these strategies are lessons learnt from UK organisations that have engaged patients across the spectrum. In a report commissioned by the UK's National Institute for Health Research to evaluate the impact and outcomes of patient engagement in funded research, one case study reported rises in recruitment rates once patient engagement officers were involved. ${ }^{7}$ Allowing patients to participate in all or most stages of the planning, administration and evaluation can reduce patient engagement barriers, reduce the risk of tokenism, and lead to improved quality of outcomes. ${ }^{83} 54$ The earlier the patient engagement, the greater the reduction in research 'waste' by focusing on the topics that matter to patients. ${ }^{6}$

Our work will inform patient engagement in the development of a quality standard on the transition from hospital to home in Ontario, Canada. Our intent is to engage patients throughout the development process 


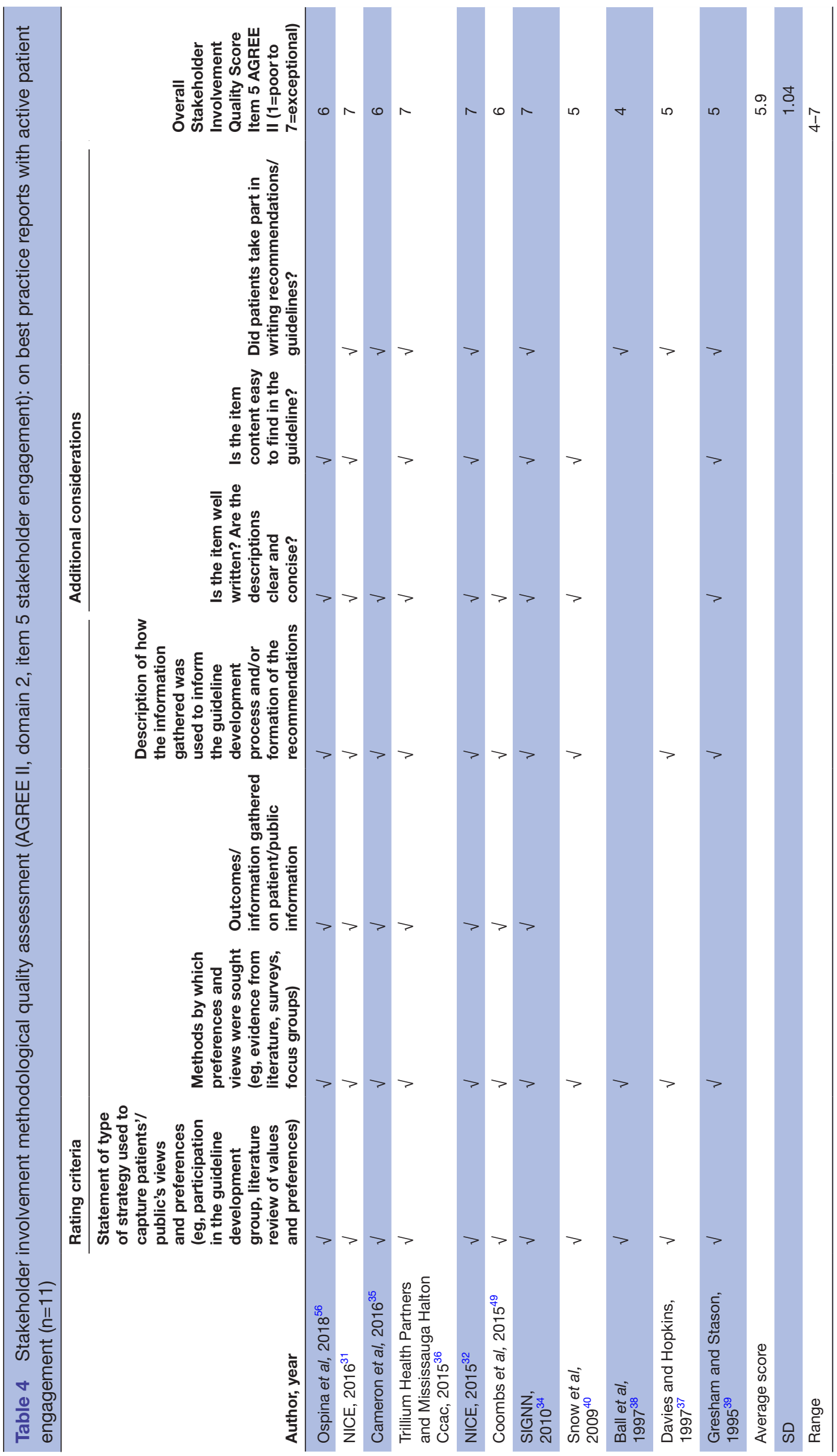


starting with a province-wide patient consultation using concept mapping to help prioritise topic areas for the standard. Lessons that we can learn from patient engagement practices in the UK include clearer documentation of patient engagement and involving patients in writing best practice recommendations.

\section{CONCLUSION}

Patient engagement has steadily increased over the last decade; however, a standardised protocol for reporting patient engagement is needed. We can learn from UK government agencies to better engage and document patient engagement as well as to consider the needs of marginalised populations in the development of best practice reports related to transitions from hospital to home. Our work will inform patient engagement in the development of a quality standard on the transition from hospital to home in Ontario, Canada.

Acknowledgements We would like to thank our information specialists from St Michael's Hospital, Nazi Torabi, Glyneva Bradley-Ridout and David Lightfoot for their expertise and support in conducting the search strategy. We would also like to acknowledge Kim Devotta for her support in facilitating this project.

Contributors Each named author has provided substantial contributions to the design, collection, analysis, drafting and revision of this manuscript. The final version was approved by all named authors. Additionally, to the best of our knowledge, the named authors have no conflict of interest, financial or otherwise.

Funding Dr. Kiran is the Fidani Chair of Improvement and Innovation at the University of Toronto. She is supported as a Clinician Scientist by the Department of Family and Community Medicine at St. Michael's Hospital and at the University of Toronto. She is also supported as an Embedded Clinician Researcher by the Canadian Institutes of Health Research and Health Quality Ontario. This research was supported by Dr. Kiran's Embedded Clinician Researcher award.

Competing interests None declared.

Patient consent for publication Not required.

Provenance and peer review Not commissioned; externally peer reviewed.

Data sharing statement The protocol and data analysis of included articles can be accessed upon request by emailing the corresponding author (grace.zhao@mail. utoronto.ca). Reuse only with permission and with citation.

Open access This is an open access article distributed in accordance with the Creative Commons Attribution Non Commercial (CC BY-NC 4.0) license, which permits others to distribute, remix, adapt, build upon this work non-commercially, and license their derivative works on different terms, provided the original work is properly cited, appropriate credit is given, any changes made indicated, and the use is non-commercial. See: http://creativecommons.org/licenses/by-nc/4.0/.

\section{REFERENCES}

1. Greene SM, Tuzzio L, Cherkin D. A framework for making patientcentered care front and center. Perm J 2012;16:49-53.

2. Mead N, Bower P. Patient-centredness: a conceptual framework and review of the empirical literature. Soc Sci Med 2000;51:1087-110.

3. Stewart M. Towards a global definition of patient centred care. BMJ 2001;322:444-5.

4. Manafo E, Petermann L, Mason-Lai P, et al. Patient engagement in Canada: a scoping review of the 'how' and 'what' of patient engagement in health research. Health Res Policy Syst 2018;16:5.

5. Domecq JP, Prutsky G, Elraiyah T, et al. Patient engagement in research: a systematic review. BMC Health Serv Res 2014;14:1-9.

6. Price A, Schroter S, Snow R, et al. Frequency of reporting on patient and public involvement (PPI) in research studies published in a general medical journal: a descriptive study. BMJ Open 2018;8:e020452.
7. Wilson P, Mathie E, Keenan J, et al. Research with patient and public involvement: a realisT evaluation - the RAPPORT study. Southampton, UK, 2015.

8. Duffett L. Patient engagement: What partnering with patient in research is all about. Thromb Res 2017;150:113-20.

9. Shamji H, Baier RR, Gravenstein S, et al. Improving the quality of care and communication during patient transitions: best practices for urgent care centers. Jt Comm J Qual Patient Saf 2014;40:319-24.

10. Richards T, Montori VM, Godlee F, et al. Let the patient revolution begin. BMJ 2013;346:f2614.

11. van der Weijden T, Pieterse AH, Koelewijn-van Loon MS, et al. How can clinical practice guidelines be adapted to facilitate shared decision making? A qualitative key-informant study. BMJ Qual Saf 2013;22:855-63.

12. Liabo $\mathrm{K}$, Boddy $\mathrm{K}$, Burchmore $\mathrm{H}$, et al. Clarifying the roles of patients in research. BMJ 2018;361:k1463.

13. Jarrett L, Unit PI. A report on a study to evaluate patient/carer membership of the first NICE Guideline Development Groups: National Institute for Clinical Excellence, 2004.

14. Schünemann HJ, Fretheim A, Oxman AD. Improving the use of research evidence in guideline development: 10 . Integrating values and consumer involvement. Health Res Policy Syst 2006;4:22.

15. Qaseem A, Forland F, Macbeth F, et al. Guidelines International Network: toward international standards for clinical practice guidelines. Ann Intern Med 2012;156:525-31.

16. Brouwers MC, Kho ME, Browman GP, et al. AGREE II: advancing guideline development, reporting and evaluation in health care. CMAJ 2010;182:E839-42.

17. Naylor MD, Aiken LH, Kurtzman ET, et al. The care span: The importance of transitional care in achieving health reform. Health Aff 2011;30:746-54.

18. Hardicre NK, Birks Y, Murray J, et al. Partners at Care Transitions (PACT) -e xploring older peoples' experiences of transitioning from hospital to home in the UK: protocol for an observation and interview study of older people and their families to understand patient experience and involvement in care at transitions. BMJ Open 2017;7:e018054.

19. Hanratty B, Holmes L, Lowson E, Eea L, et al. Older adults' experiences of transitions between care settings at the end of life in England: a qualitative interview study. J Pain Symptom Manage 2012;44:74-83.

20. Rustad EC, Furnes B, Cronfalk BS, et al. Older patients' experiences during care transition. Patient Prefer Adherence 2016;10:769-79.

21. Hvalvik S, Dale B. The Transition from Hospital to Home: Older People's Experiences. Open J Nurs 2015:05:622-31.

22. Holland DE, Mistiaen P, Bowles KH. Problems and unmet needs of patients discharged "home to self-care". Prof Case Manag 2011;16:240-50.

23. Allen J, Hutchinson AM, Brown R, et al. User Experience and Care Integration in Transitional Care for Older People From Hospital to Home: A Meta-Synthesis. Qual Health Res 2017;27:24-36.

24. Carman KL, Dardess P, Maurer M, et al. Patient and family engagement: a framework for understanding the elements and developing interventions and policies. Health Aff 2013;32:223-31.

25. CIHR. Strategy for Patient-Oriented Health Research: Patient Engagement Framework, 2014.

26. Arksey H, O'Malley L. Scoping studies: towards a methodological framework. Int J Soc Res Methodol 2005;8:19-32.

27. Tricco AC, Lillie E, Zarin W, et al. PRISMA Extension for Scoping Reviews (PRISMA-ScR): Checklist and Explanation. Ann Intern Med 2018;169:467-73.

28. Mourad Ouzzani HH, Fedorowicz Z, Elmagarmid A. Rayyan - a web and mobile app for systematic reviews. $2016 \mathrm{https} / / /$ rayyan-prod. qcri.org/.

29. Planning for effective public participation. Thornton (CO): International Association for Public Participation, 2006.

30. Brouwers MC, Kho ME, Browman GP, et al. AGREE II: advancing guideline development, reporting and evaluation in health care. CMAJ 2010;182:E839-E842.

31. NICE. Transition between inpatient mental health settings and community or care home settings: National Institue for Health and Care Excellence, 2016.

32. NICE. Transition between inpatient hospital settings and community or care home settings for adults with social care needs: National Institute for Health and Care Excellence, 2015.

33. Cowie MR, Anker SD, Cleland JGF, et al. Improving care for patients with acute heart failure: before, during and after hospitalization. ESC Heart Fail 2014;1:110-45.

34. SIGN. Management of patients with stroke: Rehabilitation, prevention and management of complications, and discharge planning, Scottish Intercollegiate Guidelines Network, 2010. 
35. Cameron JI, O'Connell C, Foley N, et al. Canadian Stroke Best Practice Recommendations: Managing transitions of care following Stroke, Guidelines Update 2016. Int J Stroke 2016;11:807-22.

36. THP and MHCCAC. Seamless Transitions: Hospital to Home Guidebook, 2015.

37. Davies E, Hopkins A. Good practice in the management of adults with malignant cerebral glioma: clinical guidelines. Working Group, Royal College of Physicians. Br J Neurosurg 1997;11:318-30.

38. Ball JW, Binder $\mathrm{H}$, Brown CW, et al. EMS for children: Recommendations for coordinating care for children with special health care needs. Annals of Emergency Medicine 1997;30:274-80.

39. Gresham GE DP, Stason WB. Post-Stroke Rehabilitation AHCPR Clinical Practice Guidelines. 16, 1995.

40. Snow V, Beck D, Budnitz T, et al. Transitions of Care Consensus Policy Statement: American College of Physicians, Society of General Internal Medicine, Society of Hospital Medicine, American Geriatrics Society, American College of Emergency Physicians, and Society for Academic Emergency Medicine. J Hosp Med 2009;4:364-70.

41. Pellett $\mathrm{C}$. Discharge planning: best practice in transitions of care. $\mathrm{Br}$ $J$ Community Nurs 2016;21:542-8.

42. Albert NM, Barnason S, Deswal A, et al. Transitions of care in heart failure. : American Heart Association, 2015:8: 384-409.

43. Australian Government. Transition care programme guidelines: Health Do, 2015.

44. RNAO Care Transitions. Registered Nurses' Association of Ontario 2014

45. Lim F, Foust J, Van Cleave J. Transitional care. Agency for health research and quality. New York: Evidence-based geriatric nursing protocols for best practice, 2012.

46. American Medical Directors Association. Transitions of care in the long-term care continuum clinical practice guideline. 2010.

47. Malcom J, Arnold O, Howlett JG, et al. Canadian Cardiovascular Society Consensus Conference guidelines on heart failure--2008 update: best practices for the transition of care of heart failure patients, and the recognition, investigation and treatment of cardiomyopathies. Can J Cardiol 2008;24:21-40.

48. Boutwell A, Bourgoin A, Maxwell J, et al. Designing and Delivering Whole-Person Transitional Care: The Hospital Guide to Reducing
Medicaid Readmissions. Rockville, MD: Agency for Healthcare Research and Quality, 2016. (Prepared by Collaborative Healthcare Strategies, Inc., and John Snow, Inc., under Contract No. HHSA290201000034I)AHRQ Publication No. 16-0047-EF.

49. Coombs MA, Darlington AS, Long-Sutehall T, et al. Transferring critically ill patients home to die: developing a clinical guidance document. Nurs Crit Care 2015:20:264-70.

50. National Health Service. The Equality Delivery System for the NHS, 2011.

51. Boaz A, Biri D, McKevitt C. Rethinking the relationship between science and society: Has there been a shift in attitudes to Patient and Public Involvement and Public Engagement in Science in the United Kingdom? Health Expect 2016;19:592-601.

52. Snape D, Kirkham J, Britten N, et al. Exploring perceived barriers, drivers, impacts and the need for evaluation of public involvement in health and social care research: a modified Delphi study. BMJ Open 2014:4:e004943.

53. Liang L, Cako A, Urquhart R, et al. Patient engagement in hospital health service planning and improvement: a scoping review. BMJ Open 2018;8:e018263.

54. Bombard Y, Baker GR, Orlando E, et al. Engaging patients to improve quality of care: a systematic review. Implement Sci 2018;13:98

55. Okrainec K, Lau D, Abrams HB, et al. Impact of patient-centered discharge tools: a systematic review. J Hosp Med 2017;12:110-7.

56. Ospina MB, Michas M, Deuchar L, et al. Development of a patientcentred, evidence-based and consensus-based discharge care bundle for patients with acute exacerbation of chronic obstructive pulmonary disease. BMJ Open Respir Res 2018;5:e000265.

57. Miller KK, Lin SH, Neville M. From hospital to home to participation: a position paper on transition planning poststroke. Arch Phys Med Rehabil 2019;100:1162-75.

58. Farrahi G. Transition of care guideline for reducing heart failure hospital readmission. Walden Dissertations and Doctoral Studies Collection 2018.

59. Dreyer T. Care transitions: best practices and evidence-based programs. , 2014:32, 309-16. 